\title{
Arrival-Time Parametric Imaging in Contrast- Enhanced Ultrasound for Diagnosing Fibrosis in Primary Biliary Cholangitis
}

Naoyuki Yoshimine

Toho University

Noritaka Wakui ( $\nabla$ wakui@med.toho-u.ac.jp)

Toho University

Hidenari Nagai

Toho University

Yoshinori Igarashi

Toho University

\section{Research Article}

Keywords: liver fibrosis, primary biliary cholangitis, shear wave elastography

Posted Date: January 4th, 2021

DOI: https://doi.org/10.21203/rs.3.rs-129916/v1

License: (a) (i) This work is licensed under a Creative Commons Attribution 4.0 International License. Read Full License

Version of Record: A version of this preprint was published at Ultrasound Quarterly on January 14th, 2022. See the published version at https://doi.org/10.1097/RUQ.0000000000000591. 


\section{Abstract}

Background: To investigate arrival-time parametric imaging (At-PI) in contrast-enhanced ultrasonography (CEUS) for diagnosing fibrosis in primary biliary cholangitis (PBC).

Methods: Participants were 48 patients (male/female: 8/40; mean age, $60 \pm 13$ years) with PBC diagnosed by liver biopsy who underwent CEUS during 2009-2019. Of these, 27 (male/female: 4/23; mean age, $61 \pm 13$ years) who also underwent shear wave elastography (SWE) were further analyzed. Sonazoid ${ }^{\mathrm{TM}}$ was intravenously injected and CEUS performed. Contrast dynamics of hepatic segment $\mathrm{V}$ and the right kidney were recorded. At-PI were generated from the recorded video clips. Contrast arrival time $<5 \mathrm{~s}$ was displayed in red, and the ratio of red (ROR) area to the entire liver contrast-enhanced area was calculated. ROR and shear wave velocity (Vs) measured by SWE were compared by fibrosis stage (F0-F3), bile duct loss score, cholangitis activity, hepatitis activity (HAO-HA3), and disease stage (stage 14), as determined by liver biopsy.

Results: ROR significantly differed between F0 and F2-F3 and between F1 and F2-3. Using ROR to diagnose ${ }^{3} \mathrm{~F} 1\left({ }^{3} \mathrm{~F} 2\right)$, area under the receiver operating characteristic curve (AUROC) was $0.77(0.92)$ (cutoff, 36.7\% [47.1\%]; sensitivity, 0.75 [0.92]; specificity, 0.82 [0.81]). Vs significantly differed between F0 and $\mathrm{F} 1, \mathrm{~F} 0$ and F2-3, and $\mathrm{HAO}$ and HA1. For diagnosing ${ }^{3} \mathrm{~F} 1$ ( $\left.{ }^{3} \mathrm{~F} 2\right)$, Vs had AUROC of 0.84 (0.80) (cutoff, $1.23 \mathrm{~m} / \mathrm{s}$ [1.40 m/s]; sensitivity, 0.75 [0.60]; specificity, 0.82 [0.86]).

Conclusions: At-PI was useful for diagnosing fibrosis, especially F2 or worse, in PBC, suggesting that AtPI can correctly diagnose fibrosis regardless of hepatic inflammation.

\section{Background}

Primary biliary cholangitis (PBC) is a chronic progressive cholestasis that affects primarily middle-aged women. ${ }^{1,2}$ The precise causes of PBC are not known, but genetic and environmental factors are likely involved and an autoimmune mechanism is implicated in the pathology. ${ }^{3}$ Prognosis of PBC is associated with fibrosis stage, ${ }^{4,5}$ which is usually determined by histopathology of liver biopsy specimens. However, histopathological diagnosis using conventional assessment systems (e.g., the Scheuer scoring system ${ }^{6}$ ) is associated with sampling errors due to heterogenous intrahepatic changes in PBC. Thus, Nakanuma et al. recently proposed a new histological staging and grading system (the Nakanuma classification ${ }^{7}$ ). Nevertheless, biopsy is an invasive procedure, and noninvasive prediction of liver fibrosis would be of great clinical utility.

Previously, we developed a novel contrast-enhanced ultrasound (CEUS) technique for quantitatively assessing changes in hepatic blood flow from portal venous dominant to arterial dominant and reported its efficacy for assessing liver fibrosis in chronic hepatitis $\mathrm{C}$ infection. ${ }^{8}$

Here, we analyzed the findings of this technique against pathohistological findings by the Nakanuma classification to determine whether this technique can diagnose liver fibrosis in PBC. 


\section{Methods}

\section{Enrollment of patients}

Subjects were patients with PBC scheduled to undergo liver biopsy at Toho University Omori Medical Center between 2009 and 2019. Written consent was provided by the patient, or by a family member if the patient was unable to do so. Exclusion criteria were (1) current or past history of hepatitis C or hepatitis B; (2) regular daily alcohol intake of $\geq 80 \mathrm{~g}$ ethanol; (3) liver tumor or portal vein embolism; (4) egg yolk allergy; (5) pregnant and/or breastfeeding; (6) age 16 years or younger; (7) severe heart disease; and (8) severe pulmonary disease. Patients with regular daily alcohol intake were excluded because its potential influence on the findings of this study was not known. Patients with liver tumor or portal vein embolism were excluded because of the possible influence on the contrast agent arrival time. Exclusion criteria 4-8 were contraindications of Sonazoid ${ }^{\mathrm{TM}}$.

This prospective study was conducted in accordance with the Declaration of Helsinki and was approved by the ethics committee at our institution (Nos. 21-26, 26-227, M18272).

\section{Contrastenhanced ultrasonography}

Ultrasonography (US) was carried out using a Canon Aplio XG (SSA-790A; Canon Medical Systems, Tochigi, Japan) with a 3.75-MHz convex array probe (PVT-375BT; Canon Medical Systems). CEUS was performed at a right intercostal space. The mechanical index was $0.22-0.29$ and the and frame rate was 15-18 frames/s. Analyzed images showed the right kidney and liver parenchyma of the right hepatic lobe (segment $₫$ ). Focus was set to $6-8 \mathrm{~cm}$ to cover the whole kidney. Patients fasted overnight before the CEUS examination. During the examination, participants were supine with the right arm above the head and held their breath. The recommended dose of perflubutane ${ }^{9}\left(0.015 \mathrm{~mL} / \mathrm{kg}\right.$; Sonazoid ${ }^{\mathrm{Tm}} ; \mathrm{GE}$ Healthcare, Oslo, Norway) was administered as a bolus via the median cubital vein at $1 \mathrm{~mL} / \mathrm{s}$ and flushed with $10 \mathrm{~mL}$ normal saline. Cine acquisition was started at the beginning of saline flush. Data generated for the first $40 \mathrm{~s}$ were saved as raw data. An independent ultrasonographer with over 26 years of experience performed US examinations and was blinded to patients' clinical characteristics.

\section{Arrival-time parametric imaging}

The ultrasound system software generated At-PI images from stored video. By simply selecting the renal parenchyma as the region of interest, the system set the point at which $80 \%$ of the region of interest was contrasted as time 0 and sequentially calculated the arrival time in individual pixels of the hepatic parenchyma. A color map is superimposed on a B-mode image. The difference in arrival time between arterial and subsequent portal venous blood to the liver is reported as $5 \mathrm{~s} .{ }^{10}$ Arrival times 0 to $<5 \mathrm{~s}$ are shown as red ( $a n d \geq 5$ to $10 \mathrm{~s}$ as yellow, indicating liver parenchyma supplied via the arterial and portal venous routes, respectively. 


\section{Measurement of contrasted areas}

Obtained At-PI data were evaluated quantitatively, as previously described. ${ }^{11}$ Briefly, the ratio of red pixel area (shorter arrival times) to the area of all contrast-enhanced pixels was calculated as the ratio of red (ROR), using ImageJ version 1.42 (National Institutes of Health, Bethesda, MD). Higher ROR indicates that the contrast-agent arrival time in the liver is closer to that in the kidney, meaning a wider area of the liver parenchyma received the contrast agent from the arterial route, indicating an arterial-portal shift in the blood flow balance toward arterial dominance in the liver. Two physicians calculated the ROR and were trained on use and interpretation of contrast agents in the liver.

\section{Shear wave elastography}

Virtual Touch ${ }^{\text {TM }}$ quantification (VTQ; Siemens Healthineers AG, Erlangen, Germany) has been used for acoustic radiation force impulse-based shear wave elastography (SWE) in our hospital since October 2012. VTQ elastography was therefore performed as additional analysis in all consenting patients. Shear wave velocity (Vs) was measured 6 times with an ACUSON S2000 US system (Siemens Healthineers AG), and the median value $(\mathrm{m} / \mathrm{s})$ was calculated. ${ }^{12-14}$ We analyzed the correlation between median $\mathrm{Vs}$ and ROR range.

\section{Serological markers}

Blood samples collected within 3 day before US examinations were used to analyze the correlation between the ROR range and various serological markers (aspartate aminotransferase [AST], alanine aminotransferase [ALT], total bilirubin [T-Bil], direct bilirubin [D-Bil], alkaline phosphatase [ALP], gammaglutamyltransferase [GGT], albumin, total cholesterol [TC], platelet count [PLT], immunoglobulin M [IgM], and fibrosis-4 [FIB-4], and AST/ALT ratio). The indirect fibrosis marker FIB-4 (age [years] × AST [U/L] / platelets $\left.\left[10^{9} / \mathrm{L}\right] \times(\mathrm{ALT}[\mathrm{U} / \mathrm{L}] / 2)\right)$ was calculated. ${ }^{15}$ The AST/ALT ratio was examined because it is reported to be associated with progression of chronic liver disease ${ }^{16}$ and $\mathrm{PBC}^{17}$.

\section{Liver biopsies}

After CEUS, liver biopsies were obtained using a semiautomatic biopsy instrument with a 16-gauge liver biopsy needle (Core II ${ }^{\mathrm{TM}}$; InterV Clinical Products, Dartmouth, MA). Specimens obtained from the anterior segment of the right lobe under US guidance were fixed in 10\% formalin, embedded in paraffin, sectioned, and stained with hematoxylin-eosin and azan for histologic evaluation. All liver specimens contained at least 15 portal tracts, including 11 complete portal triads.

The Nakanuma classification was used for histopathological assessment. ${ }^{7}$ Fibrosis (F0-F3, item A) was assessed as follows: F0, almost no fibrosis or fibrosis in the portal tracts only; F1, fibrosis extending beyond the portal area, sometimes including incomplete septal fibrosis; F2, completely connected septal 
fibrosis or bridging fibrosis with variable lobular distortion; and F3, cirrhosis (extensive fibrosis with regenerative nodules). Next, bile duct loss (BL0-BL3, item B) was assessed. Then, disease stage (Stage 14) was determined based on the combined score of items $A$ and $B$. We also evaluated cholangitis activity (CAO-CA3) and hepatitis activity (HAO-HA3) scores.

A single experienced pathologist blinded to patients' clinical characteristics evaluated all biopsy specimens. Liver biopsy and US imaging were performed on the same day.

\section{Liver pathological factor}

The ROR and Vs values obtained by US were analyzed with respect to liver pathological factors (fibrosis, bile duct loss, CA, HA, and disease stage).

\section{Statistical analysis}

Parameter analysis of ROR and Vs values was performed for each liver pathological factor; box plots were used to analyze the ROR distribution and Vs values according to liver pathological factors. The Jonckheere-Terpstra test was used to evaluate trends, and the Kruskal-Wallis test was used for comparisons between groups. Receiver operating characteristic (ROC) curves were used to assess the diagnostic performance of ROR and Vs and to determine the optimum cutoff value and area under the receiver operating characteristic curve (AUROC) for diagnosing liver pathological factors. The correlation of ROR with Vs value and serological markers was analyzed using spearman rank correlation coefficients. Logistic regression analysis was used to identify factors associated with progression of liver fibrosis. All data analyses were performed using tata version 15 (StataCorp., College Station, TX). A pvalue less than 0.05 was considered statistically significant.

\section{Results}

\section{Patient characteristics}

This study enrolled 56 patients who consented to participate. After excluding 8 patients $(2$ with a disease other than PBC diagnosed by histopathology, and 6 who could not adequately hold their breath during At$\mathrm{PI}), 48$ patients comprising 8 men and 40 women aged $60 \pm 13$ years were included in the analysis. Fibrosis stage was 0 in 18 patients, 1 in 18 patients, 2 in 10 patients, and 3 in 2 patients. Clinical, biochemical, and histological characteristics of the patients with PBC $(\mathrm{N}=48)$ analyzed in this study are summarized in Tables 1-4.

Table 1 Clinical and biochemical characteristics of 48 patients with PBC 


\begin{tabular}{|ll|}
\hline Variable & Value$^{*}$ \\
\hline Sex $(\mathrm{M} / \mathrm{F})$ & $8 / 40$ \\
\hline Age (years) & $61 \pm 14$ \\
\hline AST (U/L) & $45 \pm 21$ \\
\hline ALT (U/L) & $43 \pm 23$ \\
\hline Alb (g/dL) & $3.9 \pm 0.4$ \\
\hline T-Bil (mg/dL) & $0.7 \pm 0.3$ \\
\hline D-Bil (mg/dL) & $0.2 \pm 0.1$ \\
\hline ALP (U/L) & $504 \pm 371$ \\
\hline GGT (U/L) & $196 \pm 182$ \\
\hline TC (mg/dL) & $196 \pm 44$ \\
\hline PLT ( $\left.\times 10^{4} / \mu L\right)$ & $21.5 \pm 7.8$ \\
\hline PT (\%) & $106 \pm 22$ \\
\hline IgM (mg/dL) & $351 \pm 251$ \\
\hline FIB-4 & $2.37 \pm 7.8$ \\
\hline AST/ALT ratio & $1.14 \pm 0.38$ \\
\hline
\end{tabular}

Alb, albumin; ALP, alkaline phosphatase; ALT, alanine aminotransferase; AST, aspartate aminotransferase; D-Bil, direct bilirubin; FIB-4, Fibrosis-4; GGT, y-glutamyl transferase; IgM, immunoglobin M; PLT, platelet count; PT, prothrombin time; T-Bil, total bilirubin; TC, total cholesterol.

*Values are expressed as the mean \pm standard deviation or numbers of patients.

Table 2 Clinical and biochemical characteristics by liver fibrosis stage 


\begin{tabular}{|c|c|c|c|}
\hline Variable & F0 & F1 & $\mathrm{F} 2-3$ \\
\hline $\operatorname{Sex}(M / F)$ & $2 / 16$ & $3 / 15$ & $3 / 9$ \\
\hline Age (years) & $52 \pm 13$ & $63 \pm 14^{*}$ & $70 \pm 6^{\star \star}$ \\
\hline AST (U/L) & $39 \pm 20$ & $41 \pm 16$ & $62 \pm 22^{\star}+$ \\
\hline $\operatorname{ALT}(\mathrm{U} / \mathrm{L})$ & $40 \pm 19$ & $37 \pm 14$ & $57 \pm 34$ \\
\hline Alb (g/dL) & $4.0 \pm 0.3$ & $4.0 \pm 0.3$ & $3.6 \pm 0.5^{\star}$ \\
\hline T-Bil (mg/dL) & $0.7 \pm 0.2$ & $0.6 \pm 0.1$ & $1.0 \pm 0.3^{\star} \ddagger$ \\
\hline D-Bil (mg/dL) & $0.1 \pm 0.03$ & $0.1 \pm 0.02$ & $0.3 \pm 0.2^{\star \star} \neq$ \\
\hline $\operatorname{ALP}(\mathrm{U} / \mathrm{L})$ & $486 \pm 266$ & $460 \pm 247$ & $598 \pm 608$ \\
\hline GGT (U/L) & $154 \pm 127$ & $175 \pm 152$ & $288 \pm 263$ \\
\hline $\mathrm{TC}(\mathrm{mg} / \mathrm{dL})$ & $207 \pm 39$ & $188 \pm 35$ & $192 \pm 60$ \\
\hline $\operatorname{PLT}\left(\times 10^{4} / \mu \mathrm{L}\right)$ & $23.5 \pm 6.2$ & $21.9 \pm 9.2$ & $17.9 \pm 7.1^{\star}$ \\
\hline PT (\%) & $113 \pm 26$ & $103 \pm 16$ & $99 \pm 24$ \\
\hline $\operatorname{lgM}(\mathrm{mg} / \mathrm{dL})$ & $277 \pm 127$ & $396 \pm 249$ & $384 \pm 365$ \\
\hline FIB-4 & $1.53 \pm 0.9$ & $2.40 \pm 1.6$ & $3.58 \pm 1.2^{\star \star}+$ \\
\hline AST/ALT ratio & $1.06 \pm 0.45$ & $1.16 \pm 0.34$ & $1.22 \pm 0.33$ \\
\hline
\end{tabular}

Alb, albumin; ALP, alkaline phosphatase; ALT, alanine aminotransferase; AST, aspartate aminotransferase; D-Bil, direct bilirubin; FIB-4, Fibrosis-4; GGT, y-glutamyl transferase; IgM, immunoglobin M; PLT, platelet count; PT, prothrombin time; T-Bil, total bilirubin; TC, total cholesterol.

Values are expressed as the mean \pm standard deviation or numbers of patients.

Significant difference between fibrosis stages were examined by the Kruskal-Wallis test.

${ }^{*} p<0.05$ compared to F0. ${ }^{*} p<0.01$ compared to F0. $\uparrow p<0.05$ compared to F1. $\neq p<0.01$ compared to F1.

Table 3 Histological characteristics of 48 patients with PBC 


\begin{tabular}{|lc|}
\hline Variable & \multicolumn{1}{|c|}{ Value } \\
\hline Fibrosis stage & (F) \\
\hline F0 & 18 \\
\hline F1 & 18 \\
\hline F2 & 10 \\
\hline F3 & 2 \\
\hline Bile duct loss (BL) \\
\hline BL0 & 15 \\
\hline BL1 & 22 \\
\hline BL2 & 8 \\
\hline BL3 & 3 \\
\hline Cholangitis activity (CA) \\
\hline CA0 & 19 \\
\hline CA1 & 8 \\
\hline CA2 & 10 \\
\hline CA3 & 11 \\
\hline Hepatitis activity (HA) \\
\hline HA0 & 23 \\
\hline HA1 & 9 \\
\hline HA2 & 13 \\
\hline HA3 & 3 \\
\hline Staging & \\
\hline Stage 1 & 9 \\
\hline Stage 2 & 27 \\
\hline Stage 3 & 10 \\
\hline Stage 4 & 2 \\
\hline
\end{tabular}

Table 4 Histological characteristics of 48 patients with PBC by liver fibrosis stage 


\begin{tabular}{|llll|}
\hline Variable & F0 & F1 & F2-3 \\
\hline Bile duct loss (BL) & \\
\hline BL0 & 9 & 3 & 3 \\
\hline BL1 & 5 & 12 & 5 \\
\hline BL2 & 2 & 3 & 3 \\
\hline BL3 & 2 & 0 & 1 \\
\hline Cholangitis activity (CA) \\
\hline CA0 & 14 & 2 & 3 \\
\hline CA1 & 3 & 4 & 1 \\
\hline CA2 & 1 & 4 & 5 \\
\hline CA3 & 0 & 8 & 3 \\
\hline Hepatitis activity $(\mathrm{HA})$ & \\
\hline HA0 & 13 & 6 & 4 \\
\hline HA1 & 2 & 6 & 1 \\
\hline HA2 & 3 & 6 & 4 \\
\hline HA3 & 0 & 0 & 3 \\
\hline Staging & & & \\
\hline Stage 1 & 9 & 0 & 0 \\
\hline Stage 2 & 7 & 15 & 5 \\
\hline Stage 3 & 2 & 3 & 5 \\
\hline Stage 4 & 0 & 0 & 2 \\
\hline
\end{tabular}

\section{Correlation between At-PI and serological markers}

Correlations of ROR with AST, ALT, T-Bil, D-Bil, ALP, GGT, albumin, TC, PLT, IgM, FIB-4, and AST/ALT ratio were examined. ROR was positively correlated with AST $(p=0.004, r=0.41)$, D-Bil $(p=0.003, r=0.429)$, FIB-4 $(p<0.001, r=0.53)$, and AST/ALT ratio $(p=0.032, r=0.31)$, and negatively correlated with PLT $(p=$ $0.046, r=-0.29)$, and ALB $(p=0.002, r=-0.43)$. ROR was not significantly correlated with ALT, T-Bil, ALP, GGT, TC, prothrombin time (\%), or IgM (Table 5).

Table 5 Correlation between ROR on At-PI and serological markers 


\begin{tabular}{|lcl|}
\hline Parameter & $\mathrm{r}^{\mathrm{a}}$ & \multicolumn{1}{l|}{$p$} \\
\hline AST & .411 & .004 \\
\hline ALT & .252 & .084 \\
\hline T-Bil & .278 & .055 \\
\hline D-Bil & .422 & .003 \\
\hline Alb & -.435 & .002 \\
\hline ALP & .107 & .469 \\
\hline GGT & .163 & .270 \\
\hline TC & -.200 & .173 \\
\hline PLT & -.290 & .046 \\
\hline PT & -.252 & .084 \\
\hline IgM & .018 & .906 \\
\hline AST/ALT ratio & .310 & .032 \\
\hline Fib-4 & .526 & $<.001$ \\
\hline
\end{tabular}

Alb, albumin; ALP, alkaline phosphatase; ALT, alanine aminotransferase; AST, aspartate aminotransferase; D-Bil, direct bilirubin; FIB-4, Fibrosis-4; GGT, y-glutamyl transferase; IgM, immunoglobin M; PLT, platelet count; PT, prothrombin time; ROR, ratio of red; T-Bil, total bilirubin; TC, total cholesterol.

aspearman's correlation coefficient.

\section{Correlation between At-PI and SWE}

For additional analysis, 27 ( 4 men, 23 women) of the 48 patients consented to undergo SWE. Mean age was $61 \pm 13$ (range, 32-78) years. Fibrosis stage was F0 in 11 patients, F1 in 11, F2 in 5, and F3 in 0 . ROR range $(\%)$ was not correlated with $V s(p=0.34, r=0.19)$.

\section{Multiple comparisons of At-PI from liver pathological factors}

Fibrosis stage was F0 in 18, F1 in 18, F2 in 10, and F3 in 2 patients. Bile duct loss score was BL0 in 15, BL1 in 22, BL2 in 8, and BL3 in 3 patients. CA score was CA0 in 19, CA1 in 8, CA2 in 10, and CA3 in 11 patients. HA score was HA0 in 17, HA1 in 13, HA2 in 13, and HA3 in 3 patients. Disease stage was stage 1 in 9, stage 2 in 27, stage 3 in 10, and stage 4 in 2 patients. The Jonckheere-Terpstra test revealed that the ROR significantly increased with increasing fibrosis stage (from F0 to F3, $p<0.01$ ). Multiple comparisons showed significant differences between F0 and F2-F3 $(p<0.01)$ and F1 and F2-F3 $(p<0.01$; Fig. 1a). 
However, ROR values did not significantly differ by bile duct loss score, CA, HA, or disease stage (Fig. 1be).

\section{Multiple comparisons of SWE from liver pathological factors}

The Jonckheere-Terpstra test revealed that $V s$ values increased with the progression of fibrosis stage (from F0 to F3, p < 0.01). Multiple comparisons showed significant differences between F0 and F1 ( $p<$ 0.05), F0 and F2-F3 ( $p<0.05$; Fig. 2a), and HAO and HA1 ( $<<0.05$; Fig. 2b). However, Vs values did not significantly differ by bile duct loss score, CA, or disease stage (Fig. 2c-e).

\section{Diagnostic capability of At-PI, SWE, and serum fibrosis markers for fibrosis stage based on ROC curves}

Results of the comparison between At-PI, SWE, and serum fibrosis markers in patients with PBC are shown in Table 6.

Table 6 Results of comparison between serum fibrosis markers, SWE, and At-PI in 48 patients with PBC

\begin{tabular}{|c|c|c|c|c|c|c|}
\hline & $F \geq 1$ & & & $F \geq 2$ & & \\
\hline Method & $\begin{array}{l}\text { Sensitivity } \\
(\%)\end{array}$ & $\begin{array}{l}\text { Specificity } \\
(\%)\end{array}$ & AUROC & $\begin{array}{l}\text { Sensitivity } \\
(\%)\end{array}$ & $\begin{array}{l}\text { Specificity } \\
(\%)\end{array}$ & AUROC \\
\hline $\begin{array}{l}\text { AST/ALT } \\
\text { ratio }\end{array}$ & 0.77 & 0.50 & 0.62 & 0.67 & 0.56 & 0.60 \\
\hline FIB-4 & 0.60 & 0.94 & 0.78 & 0.83 & 0.81 & 0.85 \\
\hline SWE & 0.75 & 0.82 & 0.84 & 0.60 & 0.86 & 0.80 \\
\hline At-PI & 0.67 & 0.89 & 0.77 & 0.92 & 0.81 & 0.92 \\
\hline
\end{tabular}

At-PI, arrival time parametric imaging; AUROC, area under the receiver operating characteristic curve; FIB4, Fibrosis-4; SWE, shear wave elastography.

At-PI

The cutoff value and AUROC were respectively 47.1 and 0.92 for diagnosis of fibrosis stage F0-1 and $\geq F 2-3$, and 36.7 and 0.77 for stages $F 0$ and $\geq F 1-3$. When these values were used to diagnose cases of $\geq F 1$ and $\geq F 2$ on At-PI, the sensitivity and specificity were respectively 0.92 and 0.81 for $\geq F 2$ and 0.67 and 0.89 for $\geq F 1$ (Fig. 3a, b).

SWE

The cutoff value and AUROC were respectively 1.40 and 0.80 for diagnosis of fibrosis scores $\mathrm{F} 0-1$ and $\geq F 2-3$, and 1.23 and 0.84 for $F 0$ and $\geq F 1-3$. When these values were used to diagnose cases of $\geq F 1$ and $\geq F 2$ on At-PI, the sensitivity and specificity were respectively 0.60 and 0.86 for $\geq F 2$ and 0.75 and 0.82 for $\geq F 1$. 
The cutoff value and AUROC were respectively 2.78 and 0.85 for diagnosis of fibrosis scores $\mathrm{F} 0-1$ and $\geq F 2-3$, and 2.47 and 0.78 for $F 0$ and $\geq F 1-3$. When these values were used to diagnose cases of $\geq F 1$ and $\geq F 2$ on At-PI, the sensitivity and specificity were respectively 0.83 and 0.81 for $\geq F 2$ and 0.60 and 0.94 for $\geq \mathrm{F} 1$.

\section{AST/ALT ratio}

The cutoff value and AUROC were respectively 1.05 and 0.60 for diagnosis of fibrosis scores F0-1 and $\geq F 2-3$, and 0.96 and 0.62 for $F 0$ and $\geq F 1-3$. When these values were used to diagnose cases of $\geq F 1$ and $\geq F 2$ on At-PI, the sensitivity and specificity were respectively 0.67 and 0.56 for $\geq F 2$ and 0.77 and 0.50 for $\geq F 1$.

\section{Univariate and multivariate analysis of predictive factors for the progression of liver fibrosis in patients with PBC}

Among the four diagnostic tools for fibrosis (AST/ALT ratio, FIB-4, SWE, At-PI) that were evaluated for ability to predict progression of liver fibrosis (liver fibrosis $>F 2$ ) in patients with $\mathrm{PBC}$, multivariate analysis identified At-PI as an independent factor (Table 7).

Table 7 Predictive factors for liver fibrosis >F2 by univariate and multivariate analysis in patients with PBC

\begin{tabular}{|lllll|}
\hline & \multicolumn{2}{l}{ Univariate analysis } & \multicolumn{2}{c|}{ Multivariate analysis } \\
\hline Parameter & OR $(95 \% \mathrm{Cl})$ & $p$ & OR $(95 \% \mathrm{Cl})$ & $p$ \\
\hline AST/ALT ratio & $\begin{array}{l}2.02 \\
(0.37-11.05)\end{array}$ & .418 & & \\
& 2.21 & .005 & 1.76 & .083 \\
FIB-4 & $(1.27-3.84)$ & & $(0.93-3.32)$ & \\
& 201 & .063 & & \\
SWE & $(0.76-534)$ & & & .003 \\
At-PI & 1.11 & $<.001$ & $\begin{array}{l}1.10 \\
(1.03-1.18)\end{array}$ \\
\hline
\end{tabular}

At-PI, arrival time parametric imaging; Cl, confidence interval; FIB-4, Fibrosis-4; OR, odds ratio; PBC, primary biliary cholangitis; SWE, shear wave elastography.

\section{Discussion}

Unlike other organs, the liver has a dual blood supply, receiving about 70\%-80\% from the portal vein and about $20 \%-30 \%$ from the hepatic artery. ${ }^{18}$ Even with this dual blood supply, necrosis and desquamation 
occur in patients with chronic liver diseases such as PBC, resulting in the progression of liver fibrosis and leading to disease progression from chronic hepatitis to liver cirrhosis. As the disease progresses, the blood supply from the portal vein decreases, which is compensated by increased blood supply from the hepatic artery. ${ }^{18-21}$. In other words, the arterial-portal blood flow balance in the liver shifts from portal vein dominance to the hepatic artery dominance with disease progression. The use of imaging to quantitatively assess such changes in the balance of arterial-portal blood flow in the liver would be clinically useful as a noninvasive method for determining the severity of lesions in PBC and for predicting the development of esophageal varices and hepatocellular carcinoma.

Histopathological examination of biopsy specimens is currently required for diagnosis of PBC. However, the intrahepatic distribution of bile duct lesions is reported to be heterogenous in $\mathrm{PBC}^{22}$ which complicates the histopathological diagnosis of disease stage when a conventional scoring system is used. To address associated sampling error, the Nakanuma classification ${ }^{7}$ was proposed and is becoming a standard histopathological diagnosis system in Japan. However, the invasiveness of biopsy remains a problem. Laparoscopic examination of the liver surface was previously shown to be useful for assessing the entire liver, ${ }^{23}$ but this procedure is also invasive. Compared with liver biopsy and laparoscopy, ultrasonography is simpler, less invasive, and easier to performed repeatedly. Thus, in this study, we examined contrast-enhanced ultrasonography images, with a focus on hepatic arterialization. We showed that ROR was positively correlated with some serum fibrosis markers (i.e., FIB-4 and AST/ALT ratio). ROC curve analysis of ROR for diagnosing ${ }^{3} \mathrm{~F} 1$ and ${ }^{3} \mathrm{~F} 2$ showed AUROC of 0.77 and 0.92 , respectively, indicating that At-PI is useful for diagnosing fibrosis in PBC.

Only a few studies have investigated the noninvasive diagnosis of fibrosis in PBC. Yan et al. analyzed the results of an ultrasound-based technique (i.e., 2D shear wave elastography) against histopathological findings in 157 patients with PBC, and ROC curve analysis showed AUROC values for histopathological diagnosis of ${ }^{3} \mathrm{~F} 2,{ }^{3} \mathrm{~F} 3$, and ${ }^{3} \mathrm{~F} 4$ by the Scheuer scoring system of $0.88,0.97$, and 0.99 , respectively, indicating good diagnostic performance. ${ }^{24}$ Similarly, Park et al. examined point SWE findings against pathohistological findings in 41 patients with PBC, and showed that the AUROC values of for pathohistological diagnosis of ${ }^{3} \mathrm{~F} 2$, and ${ }^{3} \mathrm{~F} 3$ by the METAVIR classification were 0.81 , and 0.91 , respectively, indicating its good diagnostic performance. ${ }^{25}$ Vetter et al. used VTQ to perform SWE; histopathology of the liver using the Ishak score in 33 patients with PBC showed that liver stiffness increased with progression of fibrosis stage, particularly in biopsies with at least 10 portal fields $(r=$ $0.7538, p=0.0012) .{ }^{26}$ Mireen et al. performed MRI using gadolinium ethoxybenzyl diethylenetriamine pentaacetic acid as a contrast agent; histopathology of the liver showed AUROC values for ROC curve diagnosis of ${ }^{3} \mathrm{~F} 2,{ }^{3} \mathrm{~F} 3$, and ${ }^{3} \mathrm{~F} 4$ by Ludwig's classification of $0.76,0.67$, and 0.91 , respectively, indicating good diagnostic performance. ${ }^{27}$ However, none of those studies used the Nakanuma classification for histopathology, and thus, the results of those studies cannot be compared directly with the results of the present study. Nevertheless, the performance of our noninvasive technique using At-PI study appeared to be comparable to those of previously reported approaches. 
SWE results have been reported to be influenced by liver inflammation. ${ }^{28-30}$ Indeed, the Vs values in the present study differed significantly according to HA, suggesting that the SWE results were affected by liver inflammation. However, ROR values did not differ significantly according to HA. It is likely that At-PI can accurately diagnose liver fibrosis regardless of liver inflammation in patients with PBC, which is in good agreement with the findings of our previous study, which examined patients with hepatitis $C{ }^{31}$

Meanwhile, Namisaki et al. showed significantly poorer prognosis of PBC in patients with liver fibrosis with Nakanuma classification ${ }^{3} \mathrm{~F} 2$ compared with those with liver fibrosis of F0-F1. ${ }^{5}$ Multivariate analysis of the four diagnostic tools (AST/ALT ratio, FIB-4, SWE, At-PI) for liver fibrosis ( ${ }^{3} \mathrm{~F} 2$ ) identified At-PI as an independent factor, and indicated the possibility that At-PI is useful for diagnosing progression to liver fibrosis ( $\left.{ }^{3} \mathrm{~F} 2\right)$, which is associated with poor prognosis.

This study had several limitations. Our method might be affected by heart disease, which can alter the arrival time of contrast agent to the liver; renal disorders, which possible change US signal kinetics in the kidney; habitual heavy drinking, which possibly alters hemodynamics; and portal vein embolism, which possibly disturbs the balance between arterial and portal blood flow. Accordingly, this method is not applicable to patients with these conditions. Patients cannot be examined by this method if the right hepatic lobe is difficult to visualize by sonography (e.g., due to narrow intercostal spaces) or if they cannot hold their breath for 15-20 s. Perflubutane cannot be used in patients with egg allergy.

\section{Conclusion}

Analysis of hepatic blood flow balance using At-PI was useful in diagnosing fibrosis in patients with PBC. This approach accurately diagnosed fibrosis regardless of liver inflammation and was particularly useful for identifying patients with progression to liver fibrosis ( ${ }^{3} \mathrm{~F} 2$ by the Nakanuma classification).

\section{Abbreviations}

At-PI, arrival-time parametric imaging; ALP, alkaline phosphatase; ALT, alanine aminotransferase; AST, aspartate aminotransferase; AUROC, area under the receiver operating characteristic curve; CEUS, contrast-enhanced ultrasonography; D-Bil, direct bilirubin; FIB-4, fibrosis-4; GGT, gammaglutamyltransferase; IgM, immunoglobulin M; PBC, primary biliary cholangitis; PLT, platelet count; ROC, receiver operating characteristic; ROR, ratio of red; SWE, shear wave elastography; T-Bil, total bilirubin; TC, total cholesterol; US, ultrasonography; VTQ, Virtual Touch ${ }^{\text {TM }}$ quantification.

\section{Declarations}

\section{Ethics approval and consent to participate}

This prospective study was conducted in accordance with the Declaration of Helsinki and was approved by the ethics committee at our institution (Nos. 21-26, 26-227, M18272). Informed consent was provided by the patient, or by a family member if the patient was unable to do so. 
Consent for publication

Not applicable.

\section{Availability of data and materials}

Not applicable.

\section{Competing interests}

The authors declare that they have no competing interests.

\section{Funding}

This research received no specific grant from any funding agency in the public, commercial, or not-forprofit sectors.

\section{Authors' contributions}

Study concepts: Naoyuki Yoshimine

Study design: Noritaka Wakui

Data acquisition: Naoyuki Yoshimine, Noritaka Wakui,

Data analysis and interpretation: Hidenari Nagai

Statistical analysis: Hidenari Nagai

Manuscript preparation: Naoyuki Yoshimine

Manuscript editing: Noritaka Wakui

Manuscript review: Yoshinori Igarashi

\section{Acknowledgments}

Not applicable.

\section{References}

1. Kaplan MM, Gershwin ME. Primary biliary cirrhosis. N Engl J Med. 2005;353:1261-73.

2. Poupon R. Primary biliary cirrhosis: A 2010 update. J Hepatol. 2010;52:745-58.

3. Joshita S, Umemura T, Tanaka E, Ota M. Genetics and epigenetics in the pathogenesis of primary biliary cholangitis. Clin J Gastroenterol. 2018;11:11-8. 
4. Perez CFM, Hirschfield GM, CorpechotC, Floreani A, Mayo MJ, van der Meer A, et al. Fibrosis stage is an independent predictor of outcome in primary biliary cholangitis despite biochemical treatment response. Aliment Pharmacol Ther. 2019;50:1127-36.

5. Namisaki T, Moriya K, Noguchi R, Kitade M, Kawaratani H, Yamao J, et al. Liver fibrosis progression predicts survival in patients with primary biliary cirrhosis. Hepatol Res. 2017;47:E178-86.

6. Scheuer PJ. Classification of chronic viral hepatitis: A need for reassessment. J Hepatol. 1991;13:372-4.

7. Nakanuma Y, Zen Y, Harada K, Sasaki M, Nonomura A, Uehara T, et al. Application of a new histological staging and grading system for primary biliary cirrhosis to liver biopsy specimens: Interobserver agreement. Pathol Int. 2010;60:167-74.

8. Wakui N, Takayama R, Kanekawa T, Ichimori M, Otsuka T, Shinohara M, et al. Usefulness of arrival time parametric imaging in evaluating the degree of liver disease progression in chronic hepatitis $\mathrm{C}$ infection. J Ultrasound Med. 2012;31:373-82.

9. Moriyasu F, Itoh K. Efficacy of perflubutane microbubble-enhanced ultrasound in the characterization and detection of focal liver lesions: Phase 3 multicenter clinical trial. AJR Am J Roentgenol. 2009;193:86-95.

10. Shunichi S, Hiroko I, Fuminori M, Waki H. Definition of contrast enhancement phases of the liver using a perfluoro-based microbubble agent, perflubutane microbubbles. Ultrasound Med Biol. 2009;35:1819-27.

11. Wakui N, Nagai H, Ogino Y, Kobayashi K, Matsui D, Mukozu T, et al. Hepatic arterialization can predict the development of collateral veins in patients with HCV-related liver disease. J Ultrasound. 2018;21:301-8.

12. Friedrich-Rust M, Wunder K, Kriener S, Sotoudeh F, Richter S, Bojunga J, et al. Liver fibrosis in viral hepatitis: Noninvasive assessment with acoustic radiation force impulse imaging versus transient elastography. Radiology. 2009;252:595-604.

13. Kudo M, Shiina T, Moriyasu F, lijima H, Tateishi R, Yada N, et al. JSUM ultrasound elastography practice guidelines: Liver. J Med Ultrason (2001). 2013;40:325-57.

14. Sporea I, Bota S, Peck-Radosavljevic M, Sirli R, Tanaka H, lijima H, et al. Acoustic radiation force impulse elastography for fibrosis evaluation in patients with chronic hepatitis $\mathrm{C}$ : An international multicenter study. Eur J Radiol. 2012;81:4112-8.

15. Vallet-Pichard A, Mallet V, Nalpas B, Verkarre V, Nalpas A, Dhalluin-Venier V, et al. FIB-4: An inexpensive and accurate marker of fibrosis in HCV infection. Comparison with liver biopsy and fibrotest. Hepatol. 2007;46:32-6.

16. Giannini E, Botta F, Fasoli A, Ceppa P, Risso D, Lantieri PB, et al. Progressive liver functional impairment is associated with an increase in AST/ALT ratio. Dig Dis Sci. 1999;44:1249-53.

17. Nyblom H, Björnsson E, Simrén M, Aldenborg F, Almer S, Olsson R. The AST/ALT ratio as an indicator of cirrhosis in patients with PBC. Liver Int. 2006;26:840-5. 
18. Kleber G, Steudel N, Behrmann C, Zipprich A, Hübner G, Lotterer E, et al. Hepatic arterial flow volume and reserve in patients with cirrhosis use of intra-arterial Doppler and adenosine infusion. Gastroenterology. 1999;116:906-14.

19. Rocheleau B, Ethier C, Houle R, Huet PM, Bilodeau M. Hepatic artery buffer response following left portal vein ligation: Its role in liver tissue homeostasis. Am J Physiol. 1999;277:G1000-7.

20. Leen E, Goldberg JA, Anderson J, Robertson J, Moule B, Cooke TG, et al. Hepatic perfusion changes in patients with liver metastases: Comparison with those patients with cirrhosis. Gut. 1993;34:554-7.

21. Lautt WW. Mechanism and role of intrinsic regulation of hepatic arterial blood flow: Hepatic arterial buffer response. Am J Physiol. 1985;249:G549-56.

22. Dauphinee JA, Sinclair JC. Primary biliary cirrhosis. Can Med Assoc J. 1941;61:1-6.

23. Linder H, Dammermann R, Kloppel G. The laparoscopic staging of primary biliary cirrhosis. Endoscopy. 1977;9:68-73.

24. Yan Y, Xing X, Lu Q, Wang X, Luo X, Yang L. Assessment of biopsy proven liver fibrosis by twodimensional shear wave elastography in patients with primary biliary cholangitis. Dig Liver Dis. 2020;52:555-60.

25. Park DW, Lee YJ, Chang W, Park JH, Lee KH, Kim YH, et al. Diagnostic performance of a point shear wave elastography (pSWE) for hepatic fibrosis in patients with autoimmune liver disease. PLoS One. 2019;14:e0212771.

26. Vetter M, Kremer AE, Agaimy A, Pheifer L, Neurath MF, Siebler J, et al. How much liver tissue is required for sufficient histological staging in patients with primary biliary cholangitis? Digestion. 2020. doi: 10.1159/000507392.

27. Friedrich-Rust M, Müller C, Winckler A, Kriener S, Herrmann E, Holtmeier J, et al. Assessment of liver fibrosis and steatosis in PBC with FibroScan, MRI, MR-spectroscopy, and serum markers. J Clin Gastroenterol. 2010;44:58-65.

28. Rizzo L, Calvaruso V, Cacopardo B, Alessi N, Attanasio M, Petta S, et al. Comparison of transient elastography and acoustic radiation force impulse for non-invasive staging of liver fibrosis in patients with chronic hepatitis C. Am J Gastroenterol. 2011;106:2112-20.

29. Colombo S, Buonocore M, Del Poggio A, Jamoletti C, Elia S, Mattiello M, et al. Head-to-head comparison of transient elastography (TE), real time tissue elastography (RTE), and acoustic radiation force impulse (ARFI) imaging in the diagnosis of liver fibrosis. J Gastroenterol. 2012;47:461-9.

30. Yoon KT, Lim SM, Park JY, Sim DY, Ahn SH, Han K-H, et al. Liver stiffness measurement using acoustic radiation force impulse (ARFI) elastography and effect of necroinflammation. Dig Dis Sci. 2012;57:1682-91.

31. Wakui N, Nagai H, Matsukiyo Y, Ogino Y, Matsu D, Mukozu T, et al. Effect of hepatic inflammation in chronic hepatitis $\mathrm{C}$ infection on fibrosis assessment by arrival time parametric imaging. Ultrasound Q. 2018;34:128-32. 
Figures
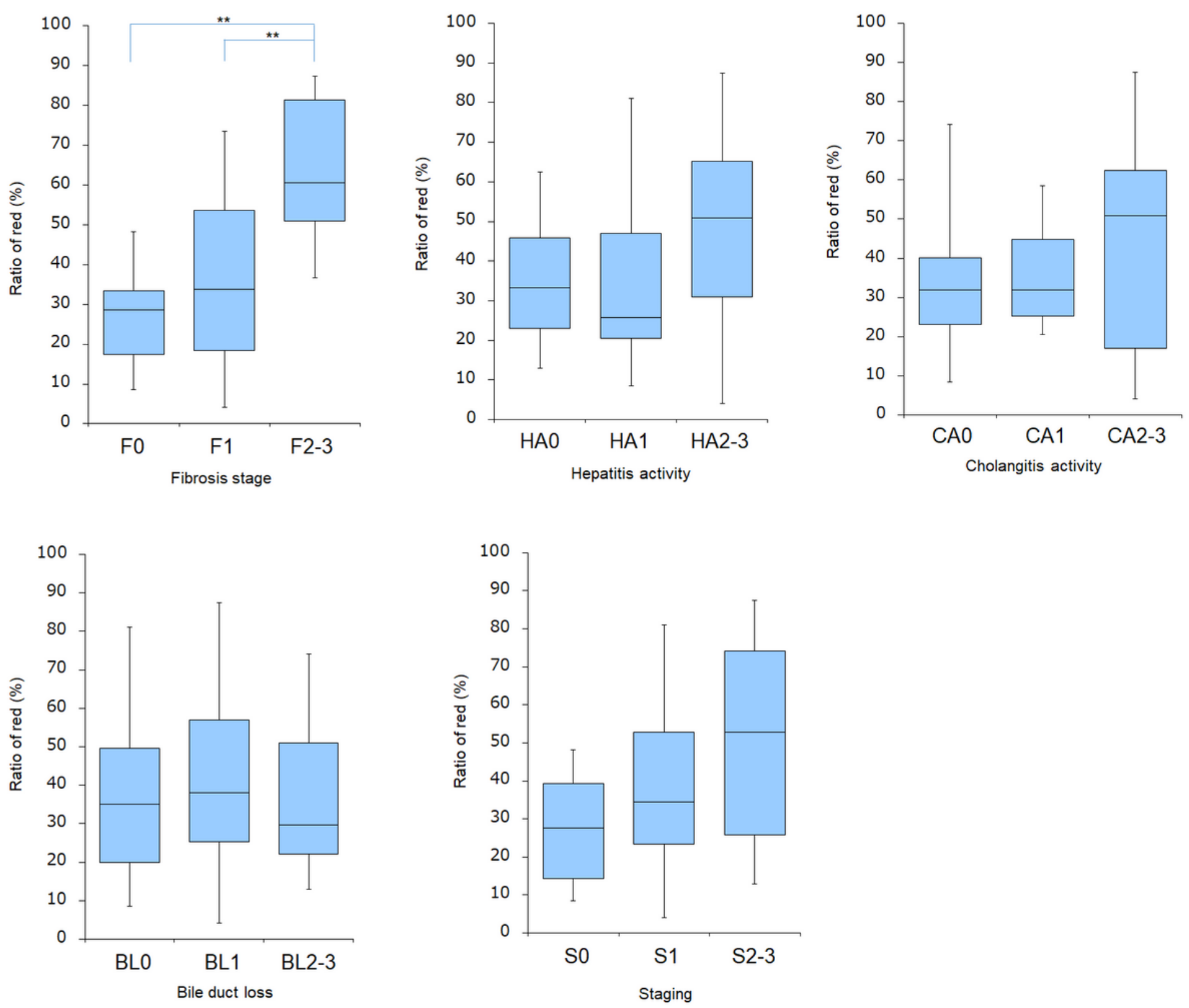

Figure 1

Multiple comparisons of At-PI with liver pathological factors (a) ROR values were significantly different between F0 and F2-F3 ( $p<0.01)$, and between F1 and F2-F3 ( $<<0.01)$. (b-e) ROR values did not differ significantly by $\mathrm{BL}, \mathrm{CA}$, and $\mathrm{HA}$ scores and disease stage. 

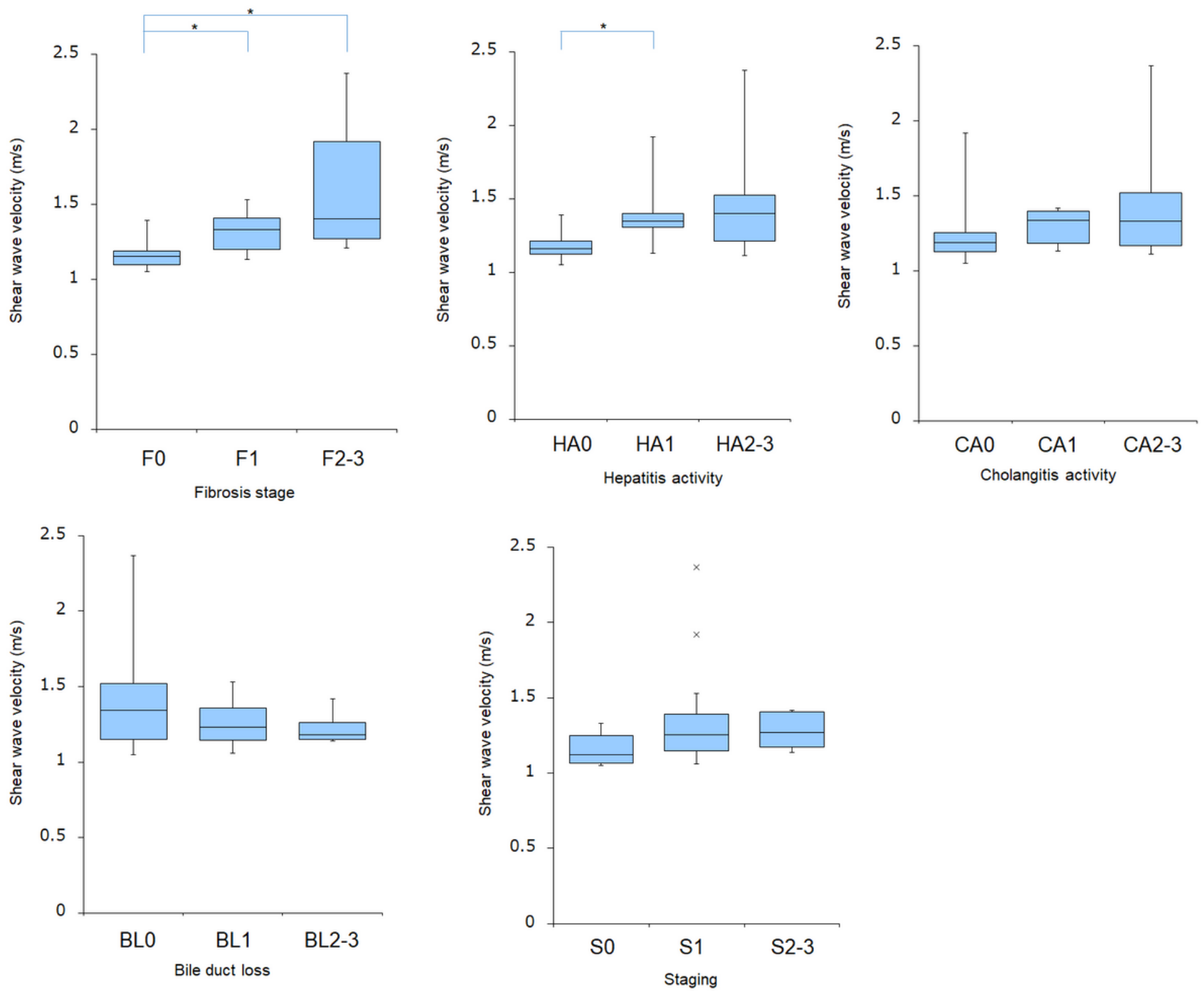

Figure 2

Multiple comparisons of SWE with liver pathological factors (a) Vs values differed significantly between $F 0$ and F1 $(p<0.05)$, and between F0 and F2-F3 ( $<<0.05)$. (b) Vs values differed significantly between HAO and HA1 ( $p<0.05)$. (c-e) Vs values did not differ significantly by BL and CA scores and disease stage. 

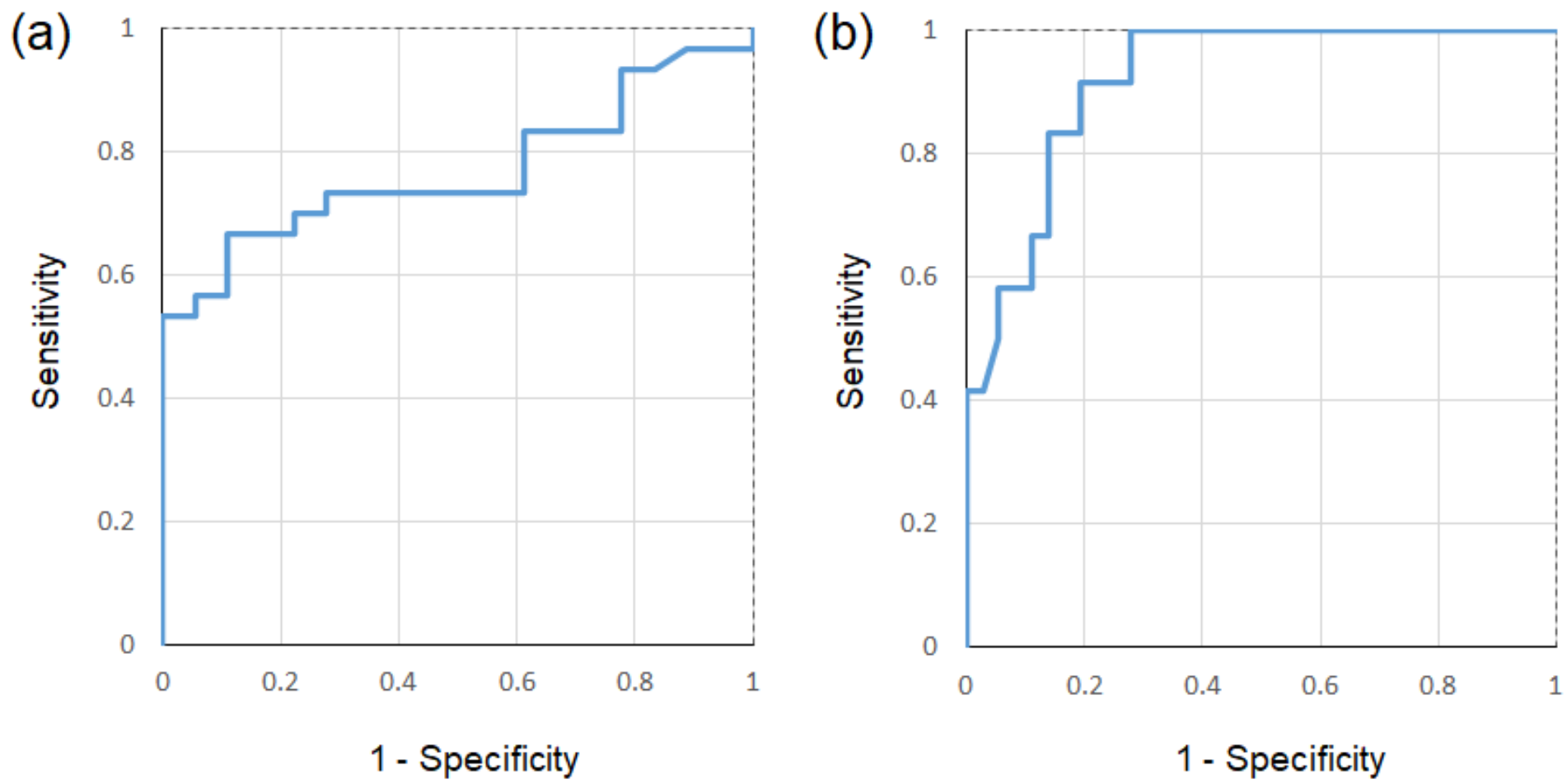

\section{Figure 3}

Capability of At-PI for diagnosing fibrosis stage based on ROC curves The cutoff value and AUROC were respectively 47.1 and 0.92 for diagnosing fibrosis stage F0-1 and $\geq F 2-3$ (b), and 36.7 and 0.77 for stages $\mathrm{F} 0$ and $\geq \mathrm{F} 1-3$ (a). When these values were used to diagnose $\geq \mathrm{F} 1$ and $\geq \mathrm{F} 2$ on At-PI, the sensitivity and specificity were respectively 0.92 and 0.81 for $\geq F 2$ and 0.67 and 0.89 for $\geq F 1$. 